\title{
Artikel
}

Nicolas Pethes*

\section{Die Semantik von ,Heimat' in Zeitschriften/Literatur des späten 19. Jahrhunderts}


Abstract: Der Beitrag fragt, auf welche Weise das Konzept von ,Heimat' in der zweiten Hälfte des 19. Jahrhunderts im zeitgenössischen Massenmedium der Zeitschrift etabliert und ausgestaltet wird. Im Rahmen des universellen Anspruchs von Rundschau- und Familienblättern kann der lokal fokussierte Heimatdiskurs als zentrierendes Element für die Miszellaneität periodischer Formate identifiziert werden. Er erlaubt es auf diese Weise auch, nach der Funktion der in diesen Zeitschriften veröffentlichten literarischen Texte zu fragen. Ein Vergleich von Adalbert Stifters kanonisierten Nachkommenschaften mit der heute vergessenen Erzählung Der Schütz' von der Pertisau von Herman Schmid, dem Herausgeber des Heimgarten, zeigt dabei, dass ,Heimat' nicht nur auf geographische und kulturelle, sondern auch auf genealogische Komplexe bezogen wird und der zugehörige Diskurs in Gestalt von Stifters Kontrafaktur des Szenarios bei Schütz auch auf ironische Zuspitzungen hinauslaufen kann - eine Tendenz, die sich allerdings nur erschließt, wenn man Literatur im Kontext ihres Erstpublikationsorts wiederliest.

The article analyzes how the concept of 'Heimat' was established and shaped in the second half of the 19 th century in the contemporary mass medium of the journal. In the context of the universal claim and the miscellaneous structure of periodical publications, the locally focused discourse on the homeland serves as a centralizing element. Based on this centripetal function, the article discusses the role of literary publications in these periodicals. A comparison of Adalbert Stifter's canonized Nachkommenschaften (Descentants) with the today unknown Der Schütz' von der Pertisau by Herman Schmid, editor of the the journal Heimgarten, shows that "Heimat" refers not only to geographical and cultural, but also to genealogical complexes. Furthermore, Stifter's satirical revision of Schmid's demonstrates that irony can also be part of he discourse on the homeland - a tendency which only becomes apparent when we analyze literary texts within the context they were first published in.

Keywords: Landschaft, Genealogie, Stifter, Journal, Journalliteratur, 19. Jh., Heimat, Stifter

*Prof. Dr. Nicolas Pethes, Universität zu Köln, Institut für deutsche Sprache und Literatur I, Neuere deutsche Literaturwissenschaft, E-Mail: npethes@uni-koeln.de

I

In nur vermeintlichem Kontrast zu seiner auf den ersten Blick klar begrenzten, häuslichen und familiären Semantik ist der Begriff der Heimat ein Produkt der massenmedialen Kultur des 19. Jahrhunderts, also des Zeitalters der Zeitschrift. Das bedeutet, dass diese Semantik in ihrer Orientierung an Natürlichkeit, Regionalismus und konservativen Werten zwar als Gegenentwurf zur Welt modernen Kommunikation- und Publikationstechnologien etabliert wurde (Sieferle 1984), diese Etablierung aber zugleich eben diesen Technologien und Medien verdankt. Denn wie Christof Hamann gezeigt hat, können Begriffe bzw. die Vorstellungen, mit denen sie in Bezug gebracht werden sollen, in periodischen Druckformaten besonders gut immer wieder in rascher Folge und in unterschiedlichen Kontexten so auf vergleichbare Weise verwendet bzw. aufgerufen werden, dass sich ihre für den aktuellen gesellschaftspolitischen Diskurs gültigen und relevanten Bedeutungen herausbilden und durchsetzen können (Hamann 2014, 9-29). Es ist mit anderen Worten gerade die inhaltliche und zeitliche Dispersität von Zeitschriften - ihre Miszellaneität und ihre Serialität (Mussell 2015, 343-358) -, die durch Redundanz und Wiederholung von Elementen in unterschiedlichen Kontexten und zu aufeinanderfolgenden Zeitpunkten zur Konzentration, Homogenisierung und Standardisierung von Begriffen wie ,Heimat' geführt hat.

Der Heimatbegriff kann in diesem Zusammenhang als Komplementärphänomen zum Konzept der Nation betrachtet werden, für das Benedict Anderson gezeigt hat, wie die fehlende Einheit politischer Gruppen über die Synchronisation von deren Weltbild durch Zeitungen sowie das Einüben des Zusammendenkens von simultanen Ereignissen durch Romane hergestellt bzw. behauptet 
wurde (Anderson [1983] 2006, 22-26). Auf vergleichbare Weise, so soll im folgenden deutlich werden, speist sich auch die einheitsstiftende Vorstellung von ,Heimat' gleichermaßen aus periodischen Massenmedien und literarischen Erzähltexten. Hinzu kommt, dass ,Heimat' wie ,Nation' ein Begriff ist, dessen gemeinschaftsstiftende Funktion aus der Konstatierung eines gemeinsamen Ursprungs der Leserinnen und Leser der betreffenden Zeitungen bzw. Zeitschriften und Romane bzw. Erzählungen resultiert - wobei die Unterschiede beider Begriffe nicht nur im Umfang der adressierten Gruppe liegen, sondern insbesondere in der mehr oder weniger expliziten Gewichtung der politischen Funktion der jeweiligen Gemeinschaftsbildung. Als solche diskursiven Konstruktionen eines gemeinsamen Ursprungs teilen ,Nation' und ,Heimat' aber eine spezifische metaleptische Funktionsweise, indem sie beide Vorstellungen repräsentieren, die von den betreffenden Diskursen mit der Behauptung generiert werden, sie lägen diesen Diskursen selbst bzw. den medialen Formaten, in denen sie artikuliert werden, immer schon zugrunde: Auch die Semantik von ,Heimat' wird in Zeitschriften in der zweiten Hälfte des 19. Jahrhunderts nachträglich zum angeblichen ursprünglichen Bezugspunkt der betreffenden Organe erklärt, den diese Organe lediglich abzubilden und in Erinnerung zu bewahren behaupten, während sie inn de facto allererst konstruieren und etablieren (Gebhard/Geisler/Schröter 2007, 9-56; Nitzke 2018, 199-214). ${ }^{1}$

Diese metaleptische Logik von Ursprungsbegriffen liegt sowohl der Erfindung des modernen Nationalismus als auch derjenigen des (nicht minder modernen) Heimatdiskurses mit seinen Spielarten der Volkstümlichkeit, seinen Artikulationsformen wie der Dorfgeschichte und den einschlägigen Bewegungen wie der Ökologie zugrunde (Lipp 1986, 331-355; Twellmann 2019; Adam 1998, 20-48; Radkau 2011). Und alle diese Aspekte und Phänomene können dabei als Faktoren wie als Indikatoren des Stabilisierungsprozesses der fraglichen Semantiken in Zeitschriften des späten 19. Jahrhunderts betrachtet werden.

Im Folgenden soll dieser Zusammenhang zwischen Medien und Semantik genauer beleuchtet

1 Die Figur der nachträglichen (kulturellen) Konstruktion eines (natürlichen) Ursprungs ist auch der Kern der LéviStrauss- und Rousseau-Lektüre in Derrida 1974. werden, indem erstens nach ,Heimat' als Programm spezifischer Zeitschriften gefragt wird: In Deutschland und Österreich erscheinen Periodika, die den fraglichen Begriff ausdrücklich im Titel tragen und damit eine semantische Fokussierung vornehmen, die als solche im doppelten Sinne als zentripetale Kraft für die in ihrer Miszellanität ansonsten zentrifugal angelegten Zeitschrifteninhalte dient ${ }^{2}$ - hinsichtlich der Wiederkehr des Titelbegriffs von Nummer zu Nummer, also als erwartungsstabilisierender Redundanzeffekt auf der Ebene des Formats; und hinsichtlich der Semantik von Heimat selbst, die auch auf Gemeinsamkeit und Zentrierung zielt und dem Medium Zeitschrift so neben dem Anspruch, breit zu informieren und zu unterhalten, einen geographischen wie ideologischen Bezugspunkt verleiht.

Dieses Spannungsverhältnis zwischen dem (miszellanen) Format der Zeitschrift und der (zentrierenden) Semantik von ,Heimat' wird dann zweitens anhand des Verhältnisses literarischer Erzähltexte zu diesem Zeitschriftenformat bzw. untereinander innerhalb einer Zeitschrift weiterzuverfolgen sein: Stützen fiktionale Beiträge die fragliche Semantik der ,Heimat' oder unterlaufen sie sie in kritischer oder ironischer Weise? Und gibt es dabei einen Unterschied zwischen nachmals vergessenen und heute kanonisierten Texten, die seinerzeit noch unmittelbar nach- bzw. nebeneinander in ein und derselben Zeitschrift erschienen sind? ${ }^{3}$

\section{II}

Fragt man nach ,Heimat' als Thema und Programm von Zeitschriften in der zweiten Hälfte des 19. Jahrhunderts, so stellt man fest, dass die

2 Zur Terminologie von Zentrifugalität und Zentripetalität für die Beschreibung der simultanen Dynamik differenzierender und homogenisierender Elemente in Zeitschriften vgl. Beetham 1990, 19-32.

3 Der vorliegende Aufsatz ist im Rahmen des Teilprojekts 1 "Poetik der Miszelle" der DFG-Forschungsgruppe "Journalliteratur" entstanden und folgt dem Ansatz, die Poetik der Literatur des 19. Jahrhunderts in Relation zu den Formatbedingungen ihrer Erstpublikation in Zeitschriften zu rekonstruieren und dabei insbesondere auch auf das Nebeneinander nachmals kanonisierter und heute vergessener Texte in den einzelnen Nummern zu achten. Vgl. Kaminski/ Ramtke/ Zelle 2014; Kaminski/Ruchatz 2017; Gretz 2016a. 
für Massenmedien charakteristische Spannung zwischen ,globalem' Repräsentationsanspruch und ,lokaler' Adressierungspraxis hier bereits deutlich nachweisbar ist. Bereits das Neben- und Gegeneinander der beiden wichtigsten Formate, Rundschau- und Familienblätter, zeigt, auf welche Weise sich Zeitschriften zwischen den Polen ,Heimat' und ,Fremde' bewegen und diese jeweils auseinander ableiten - wie die populären Titel Gartenlaube auf der einen und Über Land und Meer auf der anderen Seite unmittelbar anschaulich belegen: in beiden Fällen werden Nachrichten aus aller Welt mit der häuslichen Rezeptionssituation in Deutschland in Berührung gebracht, und die jeweiligen Titelkupfer der genannten Organe, die einmal eine Familienszene und das andere Mal exotische Reiseziele zeigen, betonen nur jeweils einen dieser beiden Pole für sich (Gretz 2016b, 279-315; Stockinger 2018).

Innerhalb dieses Spektrums existieren aber auch Zeitschriften, die den Heimatbezug ausdrücklich im Titel tragen (und von dort aus Bezug auf den Rest der Welt nehmen) - in Deutschland ab 1864 Daheim (zu dessen Beiträgern u.a. Theodor Fontane gehörte), in Österreich ab 1877 die von Peter Rosegger herausgegebene Monatsschrift Heimgarten (Farkas 2000, 177-193). Letztere ist insofern von Interesse, als sich der Zeitschriftenname aus einer mündlichen gesellschaftlichen Praxis in Alpendörfern herleitet, als deren unmittelbare Fortsetzung im Medium von Schrift, Druck und Massenpresse Rosegger sein Organ vorstellt:

"Heimgarten" nennt man in verschiedenen Alpen-
gegenden jenes Haus im Dorfe, wo man des Abends
zu kleinen handlichen Arbeiten und zur Geselligkeit
zusammenkommt - wo die geistig regsamsten, erfah-
rensten, am Erzählen und Schildern Behagen findenden
Dorfbewohner - wo Leute, welche welt- und lebensklug
sind aber oder werden mögen, zu kurzweiliger, anre-
gender, belehrender Unterhaltung sich einfinden. Im
Heimgarten werden Geschichten, Sagen, Märchen, tra-
gische und heitere Begebnisse aus dem Leben erzählt,
Lieder und Balladen gesungen; aus dem Stegreife wird
gedichtet, Schwänke und Possen werden zum Besten
gegeben, oder Tagesvorkommnisse und wichtige Ereig-
nisse aus dem Dorf- und Weltleben von den Dorfweisen
besprochen. (Rosegger 1877, 2)

Rosegger benennt hier nicht nur sämtliche Unterhaltungspraktiken im Dorfgasthaus als diejenigen Textformen, die sich auch in Zeitschriften finden und in eben dieser Mischung deren heterogenes Erscheinungsbild ausmachen, sondern auch die familienblatttypische Verbindung von Lokalem und Globalem (von „Dorf- und Weltleben") als Merkmal einer Heimatzeitschrift. Als solche versteht sich seine Zeitschrift als Fortsetzung einer sozialen Praxis, ähnlich wie dies etwa auch der Titel von Karl Gutzkows Unterhaltungen am häuslichen Herd, erschienen zwischen 1852 und 1862, suggeriert.

Vergleichbares gilt aber auch für eine weitere Vorläuferzeitschrift, die bereits 1864 unter dem Titel Der Heimgarten. Ein Haus- und Volksblatt in Bildern unter Herausgeberschaft von Herman Schmid in München erschien, allerdings nur in zwei Jahrgängen und folglich weit weniger wirkmächtig als Roseggers gleichnamige Monatsschrift, die noch über seinen Tod 1918 hinaus fortgeführt wurde. Schmid war zwischen 1860 und seinem Tod 1880 regelmäßiger Beiträger der Gartenlaube und auch ansonsten mit historischen Erzählungen aus Bayern und Tirol hervorgetreten. Dieser geographische Raum umreißt auch den Bezugspunkt seines Heimgarten, der wöchentlich auf sechzehn zweispaltig bedruckten Seiten erscheint und dessen erste Nummer 1864 mit einem Widmungsgedicht eröffnet, das bereits einiges von Roseggers Übertragung des ,Heimgarten' von einer Dorfunterhaltung auf ein Zeitschriftenformat enthält.

Auch hier findet sich die Topik des Abends als Zeit der Muße und des nachbarlichen und familiären Austauschs, der aber eben nicht nur lokale Themen "am Herde deutscher Häuslichkeit", sondern "Stadt", "Reich", "ferne Länder", "Vergang'ne[] Zeit" sowie ganz allgemein "Menschenglück" und "der Erde Leid" einschließt. Die Zeitschrift wird dabei zum einen als abendlicher Gast personifiziert, der aus dem Weltgeschehen „[w]as schön und groß" ist, "treulich wählen" wird; diese Auswahl und Mischung wird auch durch der zu einem "Kränzlein" geflochtenen „Blumen" metaphorisiert, die unmittelbar auf die Bezeichnung miszellaner Textsammlungen als Anthologie, Florilegium oder Blütenlese anspielen (Abb. 1).

Auch Schmids Heimgarten entwirft das Bild der Heimat mithin im Verhältnis zur Ferne - die Zeitschrift bringt neben Darstellungen bayerischer und tirolischer Landschaften und Bräuche auch Reiseberichte aus Italien, Griechenland oder vom Mississippi und hebt dabei auch die nationalistische Dimension dieser Gegenüberstellung von Eigenem 


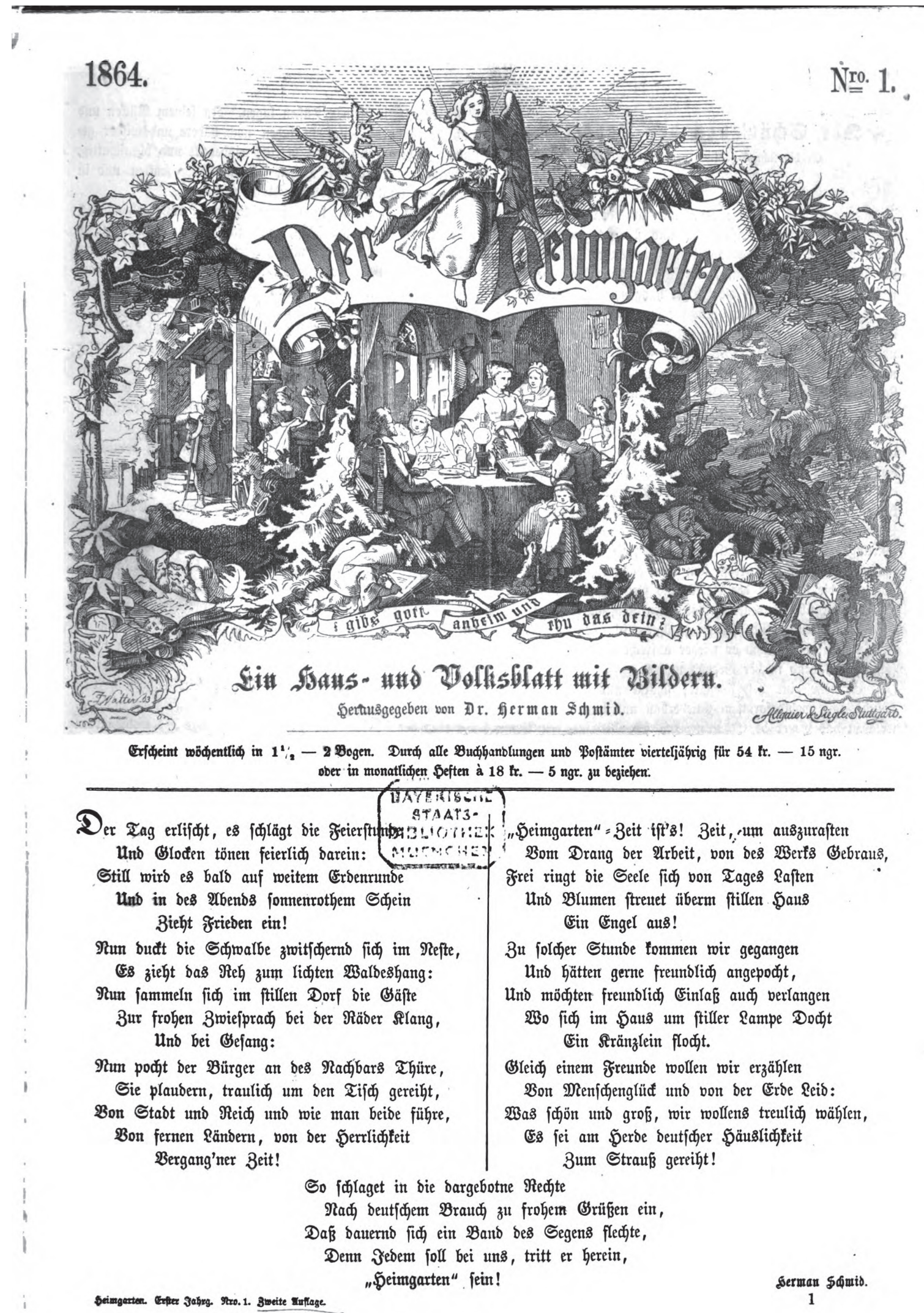

Abb. 1: Widmungsgedicht von Herman Schmid (ohne Titel). In: Der Heimgarten 1 (1864): Bayerische Staatsbibliothek München, 4 Per. 7 wp-1, Nr. 1, S. 1 urn:nbn:de:bvb:12-bsb10531387-7 
und Fremdem hervor, wenn Nr. 8 eine Karikatur zeigt, wie Germania die Engländer nach deren Protestnote im preußisch-dänischen Krieg von der politischen Bühne fegt, auf der sie einen pompösen Löwen zu geben versucht haben (Abb. 2). Auch im Rahmen einer Zeitschrift kann das Verständnis von Heimat mithin nur durch Unterscheidung oder gar polemische Abgrenzung vom Anderen konstituiert werden. Vor allem aber suggeriert die Szenerie eines alpenländischen Heimgartens bei Schmid und Rosegger, dass Heimat in erster Linie der Ort der Rezeption von Berichten aus aller Welt ist und seine Einheit also gerade aus der Gemeinschaft der Erzählenden und Hörenden bzw. Schreibenden und Lesenden gewinnt.

\section{III}

Das breite geographische Spektrum der Beiträge zu Schmids Heimgarten widerspricht also der Ausrichtung der Zeitschrift auf eine spezifische Semantik von , Heimat' gerade nicht. Das gilt auch für das breite Spektrum der Beiträge, das sich gemäß des Rundschau-Formats aus den Rubriken "Gedichte", „Erzählungen", "Geschichtliches", "Naturwissenschaftliches", "Länder- und Völkerkunde", "Vermischtes" sowie "Musik" speist. Auch hier ist es die Vorstellung eines lokalen und homogenen Rezipientenkreises, der die Divergenz und Miszellanität der unterschiedlichen Nachrichtenund Wissensbereiche bündelt.

Auffällig und ebenfalls konform mit dem Rundschau-Format ist die Vorrangstellung literarischer Beiträge, mit denen die einzelnen Hefte des Heimgarten jeweils eröffnen. Lässt man die Gedichte beiseite, versammelt der erste Jahrgang von Schmids Zeitschrift 21 Erzählungen, die meist in drei bis vier Lieferungen in Fortsetzung erscheinen, wobei nicht alle Erzählungen als Aufmacher dienen, so dass mitunter auch mehrere Serien parallel durch eine bestimmte Anzahl von Heften laufen. Einige dieser Texte sind programmatisch heimatbezogen, so etwa "Allerlei Geschichten aus Tirol" von Adolf Pichler und die erste Erzählung, die der Herausgeber Schmid selbst beisteuert, "Der Schütz' von der Pertisau". Schmids weitere zwei Erzähltexte, "Die Goldsucher" und "Mordweihnacht", tragen ihr anderes Sujet aber bereits im Titel. Und auch die übrigen Erzählungen bedienen unterschiedliche Genres, darunter zahlreiche von seinerzeit populären Familienblatt- und Romanautorinnen wie Ottilie Wildermut („Die Schule der Demuth") und Emmy von Dincklage ("Das SechsUhr-Männchen"), politischen Schriftstellern wie Ferdinand Kürnberger („Eis" und "Schulmeister Krachenberger") sowie je eine Erzählung von Felix Dahn ("Leben und Schule") und Adalbert Stifter ("Nachkommenschaften"). Insbesondere letztere sticht als nachmals kanonisierter Text aus dem Umfeld heute meist vergessener Autoren aus dem Inhaltsverzeichnis des erstens Jahrgangs hervor, erinnert aber dadurch daran, dass auch und gerade kanonische Texte der deutschsprachigen Literaturgeschichte als ursprünglichen Publikationskontext oft den einerseits heterogenen und andererseits auf ein spezifisches inhaltliches Programm ausgerichteten Zusammenhang einer Publikumszeitschrift hatten.

Nähert man sich der Frage nach diesem inhaltlichen Programm im Fall des Heimgarten über die Rubrik der literarischen Erzähltexte, so eröffnet sich auf diese Weise ein zweifacher Horizont für die Analyse: Zum einen kann man untersuchen, auf welche Weise literarische Erzähltexte an der Ausgestaltung und Etablierung, vielleicht aber auch Reflexion der spezifischen Semantik von Heimat beteiligt sind, die die Zeitschrift entwirft. Dabei geht es nicht nur um die jeweils einzelnen Erzählungen für sich genommen, wie man sie auch außerhalb des Publikationskontexts einer Zeitschrift studieren könnte, sondern auch um die spezifische Abfolge und Nachbarschaft, also das Nach- und Nebeneinander der verschiedenen literarischen und nicht literarischen Beiträge von Heft zu Heft, in einem Heft, auf einer Heftseite etc. Und aus eben dieser Perspektive ergibt sich die zweite Analyseperspektive: Denn zum anderen kann man auf diese Weise auch untersuchen, wie sich ein nachmals nur noch in kanonischen Werkausgaben interpretierter Text in seinem ursprünglichen Publikationskontext ausnimmt und welche zusätzlichen Dimensionen der Textdeutung sich aus dieser Rekontextualisierung ergeben, wenn man davon ausgeht, dass Veröffentlichungen in Zeitschriften in Wechselbeziehung zu weiteren in derselben Zeitschrift veröffentlichten Texten stehen, die bei ihrer Wiederveröffentlichung in der Sammlung oder Werkausgabe nur eines Autors zwangsläufig verloren gehen - und also wiederzuentdecken sind. 

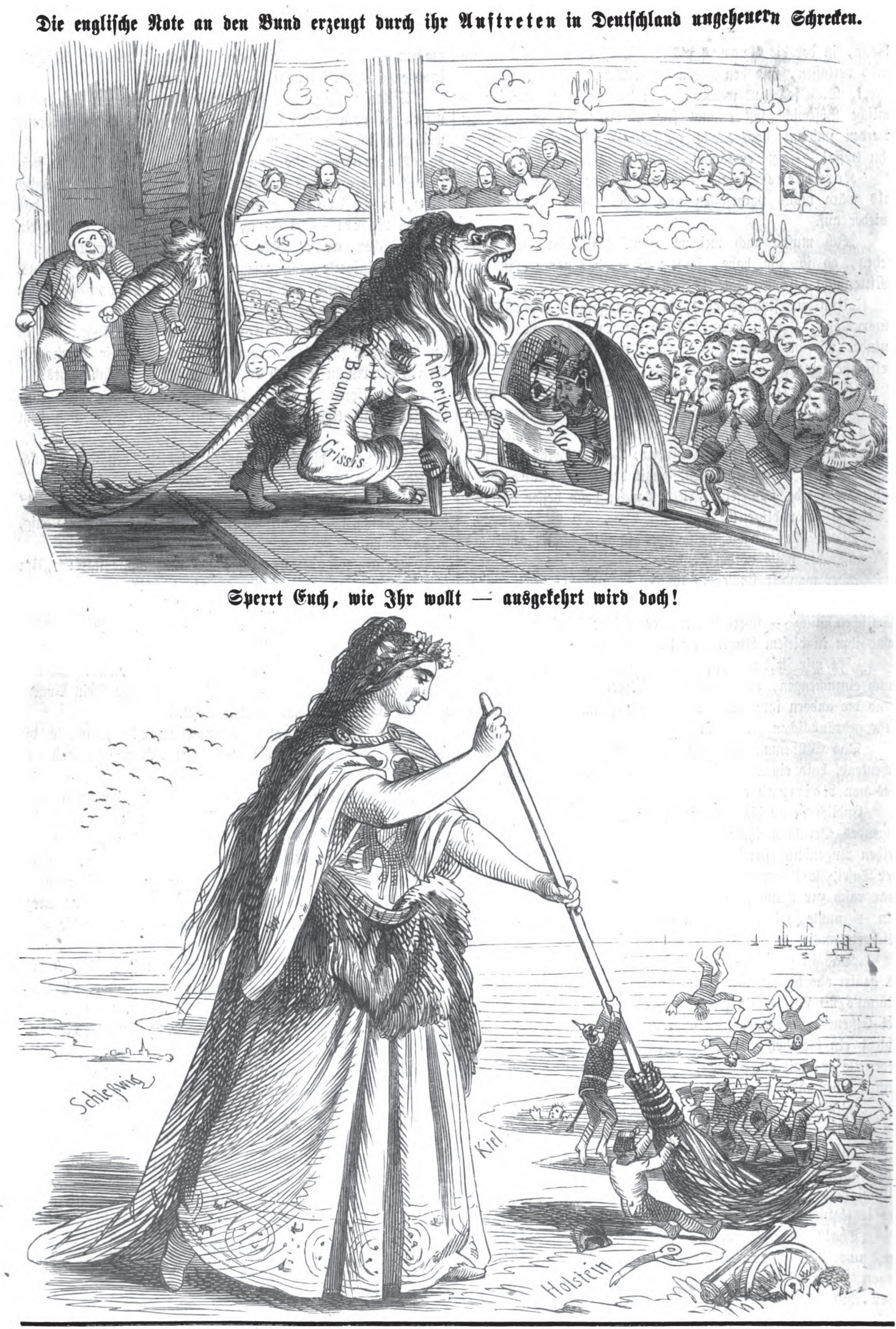

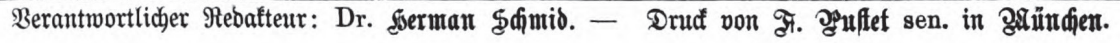

Abb. 2: Karikatur (ohne Angabe des Zeichners). In: Der Heimgarten 1 (1864): Bayerische Staatsbibliothek München, 4 Per. 7 wp-1, Nr. 8, S. 128 urn:nbn:de:bvb:12-bsb10531387-7 
Vor dem Hintergrund dieser beiden Fragen sollen nun exemplarisch die beiden zu Beginn der ersten Nummern des Heimgarten veröffentlichten literarischen Texte, die heute vergessene, damals aber als Aufmacher des Herausgebers zentral gesetzte Erzählung "Der Schütz' von der Pertisau" von Herman Schmid sowie Adalbert Stifters "Nachkommenschaften", vergleichend betrachtet werden (Abb. 3 und 4). Diese beiden Erzählungen erscheinen unmittelbar aufeinanderfolgend in den Nummern 1-5 sowie 6-8 und sind somit zum einen jeweils in sich sequentialisiert und stehen zum anderen untereinander in einem Fortsetzungsverhältnis. Zu dieser doppelten Serienstruktur kommt hinzu, dass beide Texte in den fraglichen acht Ausgaben des Heimgarten zusammen mit einer Reihe anderen Beiträge erscheinen, etwa dem meteorologischen Artikel von Rudolf Falb "Die Nächte im Heimgarten. Unterhaltungen aus der Sternenkunde" in Heft 4, einem Abriss von "Beethoven's Jugend" in Heft 6 oder den bereits erwähnten Bericht "Auf dem Mississippi" in Heft 8. Stifters "Nachkommenschaften" werden in diesem Umfeld in drei Lieferungen präsentiert, von denen die erste mit acht Seiten die Hälfte der Nr. 6 umfasst; und entsprechend ist auch die zweite Lieferung am Seitenlayout der Zeitschrift und nicht an inhaltlichen Zäsuren der Erzählung orientiert. Auf welche Weise nun lassen sich aus diesem Publikationskontext Einsichten über die gleichermaßen massenmediale wie literarästhetische Konturierung der Heimatsemantik gewinnen?

\section{IV}

Zu den Eigenheiten dieses Kontextes gehört, dass Schmids Erzählung "Der Schütz' von der Pertisau” hier kurz referiert werden muss, weil sie im Unterschied zu Stifters "Nachkommenchaften" heute vergessen ist. Die Heimatthematik wird hier im historischen Gewand einer Episode aus dem 17. Jahrhundert aufgerufen, in der Herzog Sigismund Franz von Tirol, der aufgrund seines priesterlichen Zölibats der letzte seiner Linie ist, zum Opfer einer Intrige seiner italienischen Verbündeten wird, die seinen Leibarzt unter dem Einfluss der „dämonischen Schönheit" Fiammina dazu bringen, ihn zu vergiften (Schmid 1877a, 20). Das Bild der Heimat wird dabei zum einen durch Landschaftsschilderungen aufgerufen, die zu Beginn zunächst die erhabene Gewalt eines Gewitters im Hochgebirge betreffen, in dem der Herzog auf einer Wanderung in Lebensgefahr gerät, bevor er von einer treuen Untertanin gerettet wird - eine Szenerie, die nicht nur die spätere Bedrohung seines Lebens symbolisch vorwegnimmt, sondern auch deutlich an vergleichbare Szenarien bei Stifter erinnert. Dem stehen aber vor allem die idyllischen Beschreibungen des Tiroler Berglands zur Seite:

Es ist ein wundervolles Berggelände, die Gegend am
Achen-See und manch' Einer, der die Welt diesseits
und jenseits der Meere geseh'n, mag bei seinem
Anblick bekennen, er hab in allen Landen seiner Wan-
derungen einen schönern Erdenwinkel nicht erschaut.
Wer aus den tiefen, duftig grünen Waldthälern des
bayrischen Hochlandes tritt, und an den Gebirgswäs-
sern hinaus gelangt, wo Thal und Kirche sich nach der
Ach, dem Ausflusse des See's benennen und wer dann
noch eine Strecke fortwandert an dem riesigen lang-
gestreckten Bergrücken des Unutz dahin: vor dem thut
sich, wie ein seliges Liebesgeheimniß der Bergkessel
auf mit dem schmal hinngegossenen Wasserspiegel,
dessen mährchenhafte Bläue den Azur des Himmels
beschämt. (Schmid 1877a, 17)

Zum anderen basiert die Vorstellung von Heimat auf kulturellen Eigenheiten, die dem Herzog deutlich werden, als er in einer Gesangsdarbietung Fiamminas zwar "viele Schönheit, [...] viele Kunst, viel Klang und Ausdruck" findet, zugleich aber feststellt, dass inn eine "Leere mitten in diesem Überfluß", befällt. Als Alternative zu der manierierten italienischen Kultur "verlangt" es ihn "nach deutschen Tönen", die ihn dann aus der "schlichten herzigen Weise" entgegenklingen, die seine eigenen Kammermusiker vortragen:

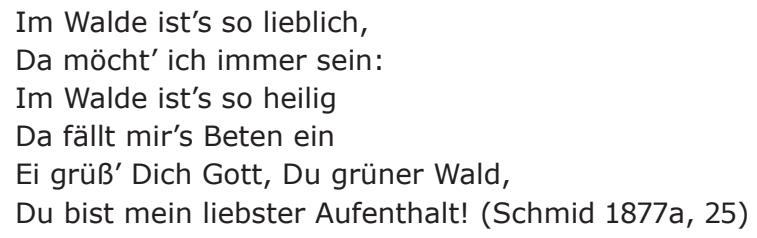

Natürlichkeit wird mithin gegen Künstlichkeit gesetzt, und der Herzog erkennt in der Folge, dass sämtliche Reize des Auslands - darunter auch das Angebot, als Statthalter Spaniens in die Niederlande zu gehen - hinter seiner Verbundenheit mit den Tiroler Landeskindern zurückstehen müssen. In Anlehnung an Jean Pauls Idyl- 


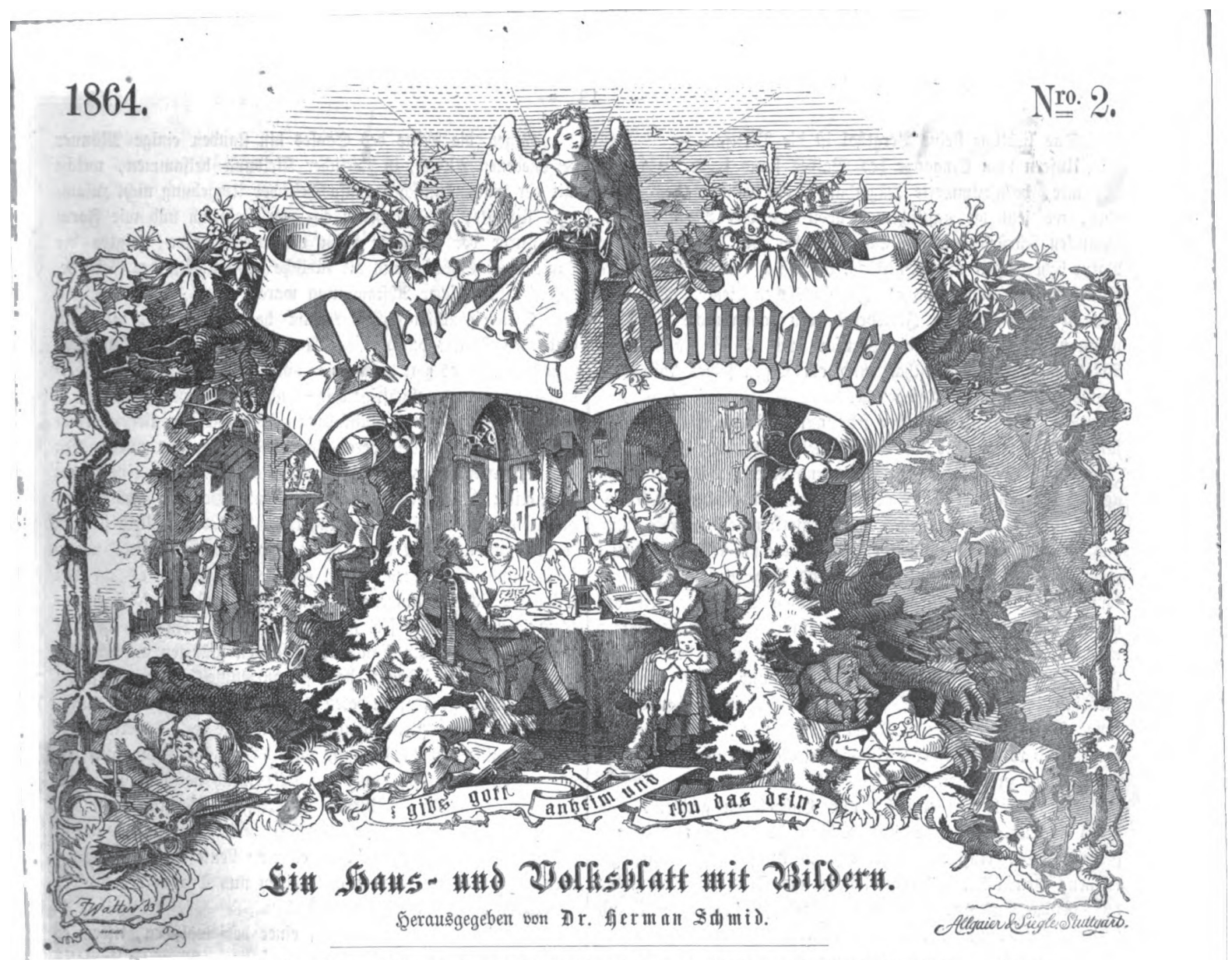

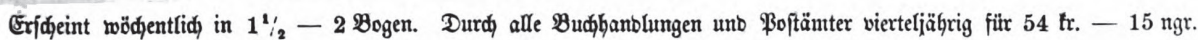
ober in monatlicen ફeften à 18 tr. $-5 \mathrm{ngr}$. zu beziehen.

\section{Der Gøüb' vou ber \$ertijau. Bejdidftlide Erzählung von ferman Sdimio. (rotticțung.)}

II.

Ess ijt ein wunbervolles Berggelände, bie Giegent am 2 Achen=

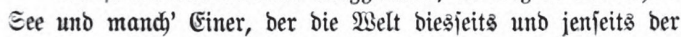
Meere gejeb'n, mag bei jeinem 2 tnblicf befennen, er habe in allen Lanben feiner $\mathfrak{B a n b e r u n g e n ~ e i n e n ~ i c j o ̈ n e r n ~ E r b e n w i n f e l ~}$ nidgt eridaut. WBer aus ben tiefen, buftig grïnen $\mathfrak{W a l b t h a ̈ =}$

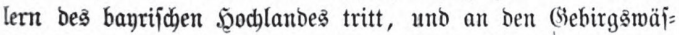
iern binaus gelangt, wo Thal uno Sirche fich nad) ber $\mathscr{H}($ d), bem $\mathfrak{A}$ usflufije bes See's benennen und wer bann nod eine Strefe fortwandert an bem riefigen langgeftreeften Bergrïfen

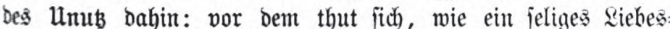
geheimniś ber Bergfeijel auf mit bem idjmal hingegoifenen

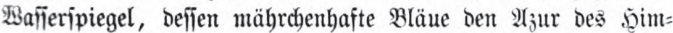
melş beichämt. Biele verbringen eine glüdtliche Etunde an bem idimalen (Bejtabe und fehren befriedigt heim, ein idjönes Bill in ber Seele, eine holde Erinnerung mehr im (Semüthe; ber reizendite 2 nnblicf aber bleibt bem vorbehalten, ber ben Rachen bejteigt uno ein paar Etunden babinrubert zwijichen ben $\mathfrak{B a l d b a b g a ̈ n g e n ~ u n d ~ b e n ~ w i b e r b a l l e n d e n ~ F e l j e n , ~ d i e ~ j t e i l ~}$ unb unwegiam in bie gllut abftürzen. Dann bebnt ber See,

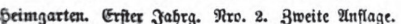

ber aus ber Ferne ftreng abgeidsloffen jocheint, fich überraidbento zu einem breiten gerunbeten 2 Sajperbecfen aus, und nad) z̧wei Éeiten hin geht ein holder, herzerfreuenber $\mathfrak{A n b l i c f}$ anj.

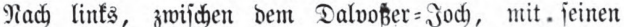
burgartig geformten Feljenziunen und zwijuen bem Mütfen bes Bfauferiteinz jenft fid ein breiter Bergeinjonnitt herab und läß̈t aus jtundenweiter Entfermung bie Berge hereinblicfen, welche bas gegenüber liegende recbte $\mathfrak{u}$ fer bes Unterinntgals begremzen und Den (Eingang in's Billertbal bewact)en. Iavor, aus grüner, baumreidjer Ébene ragt bas Sirrthlein ,in ber Eben" ber jeligen Mago Motbburga vielbejudter Mutheplab mit ipibigem Thurm empor, uno hinter bemjelben fällt ber tojende,

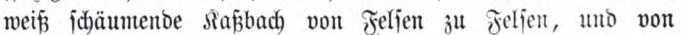
Mithblwerf zu Mühlwerf, biß himunter, wo ihn bie podyenden

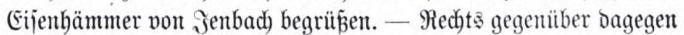
weitet fich) noch rülfwärts ein fleines Thäldben in janttem Bogen ans, won imaragbarünen 2 Seiden bebectt, hinter iweldyen' bie

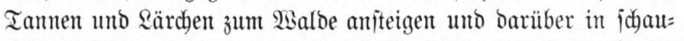
riger Eritarrung bie riejenhaften Feljemipißen bes Irijtentopfes

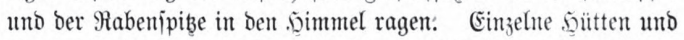
Bauernbäujer liegen zerftreut in bem traulich beidräntten Maum,

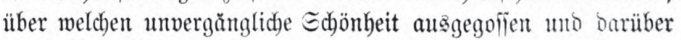
bie heilige Ruhe geiftnährender Einjamfeit gebreitet iit, wie ber Edjleier über ein holdes, ernitlächetndes Frautenantlib.

Abb. 3: Herman Schmid: „Der Schütz” von der Pertisau". In: Der Heimgarten 1 (1864): Bayerische Staatsbibliothek München, 4 Per. 7 wp-1, Nr. 2, S. 18 urn:nbn:de:bvb:12-bsb10531387-7 


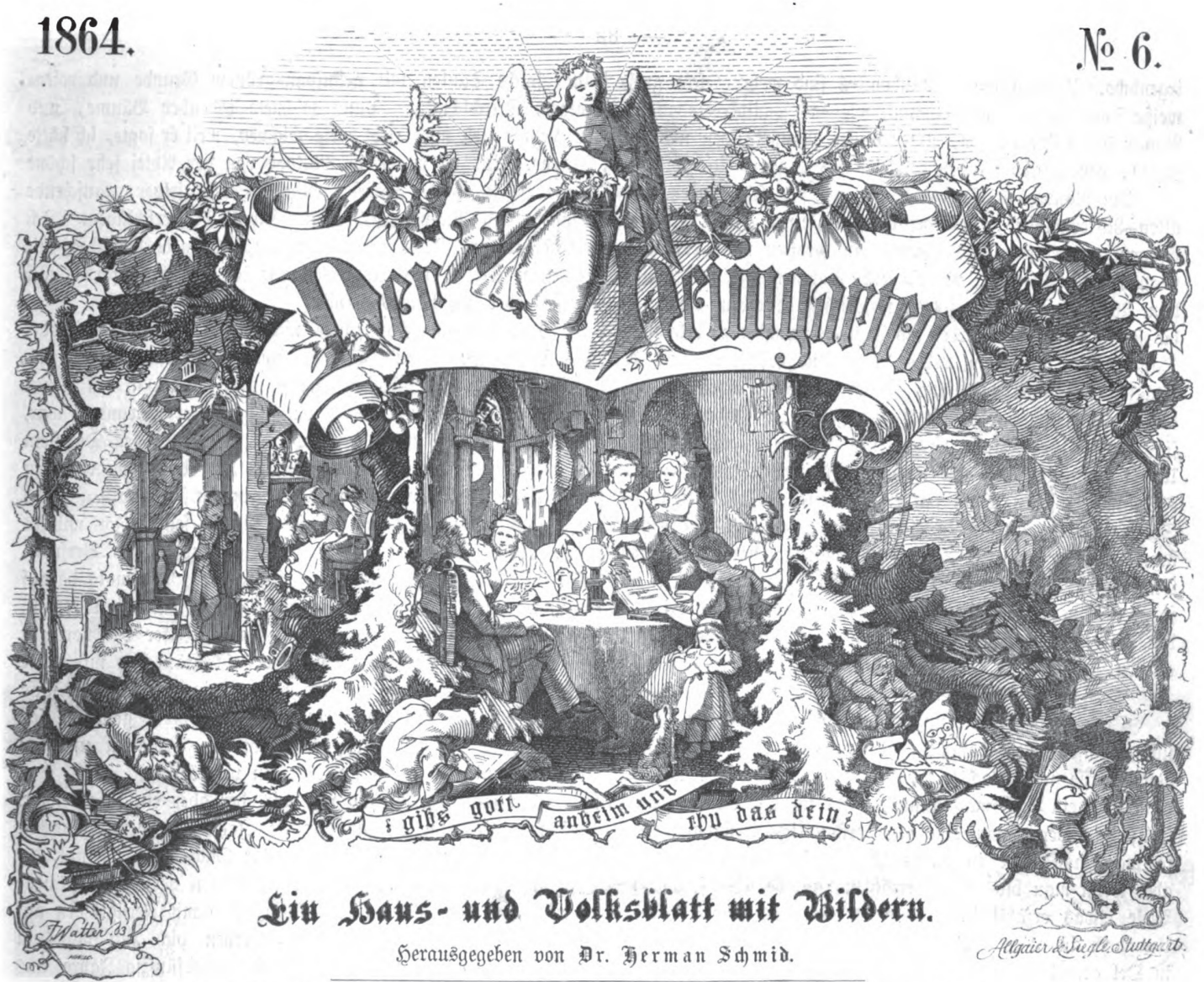

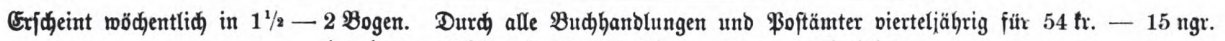
Dber in monatlidjen Şeften à $18 \mathrm{fr}$. $-5 \mathrm{ngr}$. zu beziehen.

\section{Madtommenjofiten.} Bon gicolbert \$fifter.

\$o bin id unveriehenz ein Lanbichaftamaler gemorben. Esz ift entieglich. Bent man in eine Sammluntg neuer Bilber geräth, weld') eine Menge won Lanbjidjaften gibt ez̉ ba; wenn

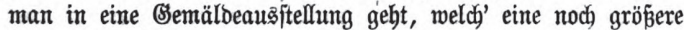
Menge von \&anbidaften trifft man ba an, und wenn man alle Sanbidaften, welche von allen \&andichaftzmalern unjerer Beit gemalt werben, von foldhen Ranbjhaftżmalern, bie ihre Bilder verfaufen wollen, unt von folden, bie ihre Bilder niddt ver faufen wollen, ausftellte, - weld' allergrö̈pte Merge von $\mathfrak{L} a n d=$

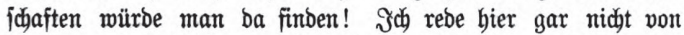
veriđämten Töbthtern, weldhe in $\mathfrak{B a f f e r f a r b e n ~ h e i m l i d ) ~ e i n e ~}$ Trauerweibe malen, unter welderer irgend ein befränzter $\mathfrak{R}$ rug

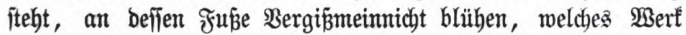
bie Mutter zum Geburtstage erbalten joll; idy rebe ferner nidht von ben Erzeugnififen, weldhe reijente Frauen ober Mäbchen von bem Dampfichiffe ober bem Fenjter ihres (șajthaujes aus in ihr Şandbuch als Erinterung eintragen; id rede aud nidyt von ben Ranbidaften, weldhe Schönichreibmeifter in ibre Ber=

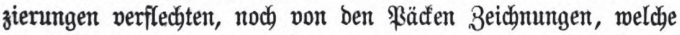
alljäbrlich in ben Fräuleinjidulen verfertiget werben, unter benen fich viele Sanbidbaften mit Bäumen befinben, auf benen Sands= Setmgarten. Erfer Sabrg. Mro. 6.

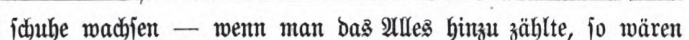

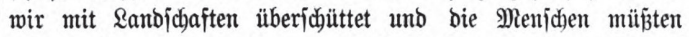
verzweifeln. $\mathfrak{R} u$, eş find ber in Delfarben gemalten und mit Bolbrabmen veriebenten Rambichaften idgon genug. Uno ich will mun audb nod, jo viele Ranbjdyaften mit Delfarben malen, als

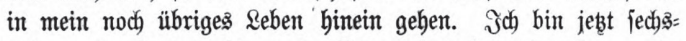

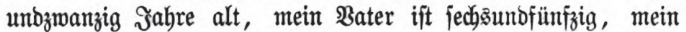
(3roppater achtundackzig, uno beibe find fo rïitig unt gejund,

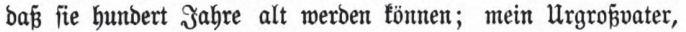

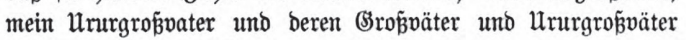

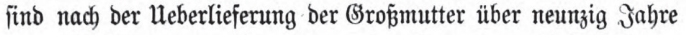

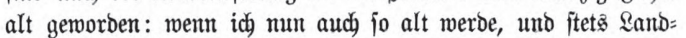
ichaften male, fo gehören, fallz idy jie alle am Seben lafife, unt ïe einmal in Rijten jammt ihren $\Re$ ahmen verpadt verführen will, fünfzebn zmeifpännige $\mathfrak{B a ̈ g e n ~ m i t ~ g u t e n ~ \Re o j i ̄ e n ~ b a z u , ~}$ woß̌ei ich nod) fo mandhen malfreien und vergrügten Tag verleben Kann.

Dả ijt betrachtenesmüroig.

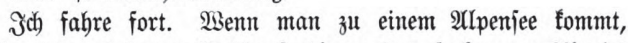
uno in einem einfamen (3ajthauje übertrachtet, fo fommen 2 (benos brei ober vier \&andichaftsmaler in bie Sajtitube, weldye unter $\mathfrak{T a g a z ~ a u f ~ v e r i d j i e b e n e n ~ S t e l l e n ~ b e s ~} \mathfrak{A}$ ngerz gejeffen fint und gemalt haben. Die fich an bem Mande bes (Sletichers befinden,

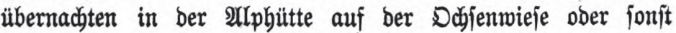

Abb. 4: Adalbert Stifter: "Nachkommenschaften", in: Der Heimgarten 1 (1864):

Bayerische Staatsbibliothek München, 4 Per. 7 wp-1, Nr. 6, S. 81 urn:nbn:de:bvb:12-bsb10531387-7 
lendefinition bekennt er sich zum Glück „in der Beschränkung" (Schmid 1877b, 36), also in der Heimat, und lässt sich von seinem Vertrauten, dem Grafen Harras, dazu bewegen, den Papst um Aufhebung des Zölibats zu bitten und eine bayerische Prinzessin zu heiraten, um den Fortbestand seines Herrschergeschlechts zu sichern. Da er aber bei der Hochzeitsfeier Opfer des erwähnten Giftattentats wird, fällt Tirol an den österreichischen Kaiser und verliert seine unter Franz Sigismund genossene Freiheit.

Die politische Konturierung von Heimat in Abgrenzung von Österreich (durch die die Geschichte Tirols mit derjenigen Bayerns als Erscheinungsort des Schmid'schen Heimgarten verbunden ist) wird mithin mit dem semantischen Feld von Natürlichkeit und Einfachheit auf der einen Seite sowie Freiheit und genealogischer Kontinuität auf der anderen Seite gekoppelt - und das, indem insbesondere letztere als bedroht und also schützenswert vorgestellt werden. Auf diese Weise lässt sich in Schmids Erzählung dasjenige Ineinander von vertikalen und horizontalen Relationen erkennen, das Christof Hamann in seiner Analyse von Kulturzeitschriften im letzten Drittel des 19. Jahrhunderts als deren Makrostruktur herausgearbeitet hat: Wie Hamann zeigt, sind die miszellanen Beiträge in den verschiedenen Zeitschriften dadurch verbunden, dass sie immer wieder dieselben vertikalen Relationen - also etwa Stadt vs. Land oder Natur vs. Kultur - aufgreifen und mit horizontalen Relationen - hier also etwa nationale Gegensätze wie Deutschland vs. Österreich oder Tirol vs. Österreich - koppeln (Hamann 2014, 33-42). Durch die Möglichkeit, dieses Relationsgefüge in verschiedenen Texten immer wieder zu wiederholen, sind es gerade Zeitschriften, die auf diese Weise ein semantisches Paradigma etablieren, und diese semantischen Paradigmen sind dabei stets als Mittelwert zwischen den möglichen Extrempolen der jeweiligen Relationen angelegt, wie Hamann in Anlehnung an die Normalismusforschung zeigen kann. Eben diese Merkmale weist auch das Heimatbild in Schmids Erzählung auf, wenn der Herzog seine Vorstellung tirolischer Schlichtheit aus der Abgrenzung gegen ,wälschen' Überfluss konturiert und dabei ein Bild der Natur evoziert, das anstelle erhabener Gipfel zunehmend Bergseen und "Waldesrauschen" in den Vordergrund rückt (Schmid 1877a, 25). Und dass dieser Befund so vertraut und kaum erwäh- nenswert wirkt, belegt gerade die Wirkmächtigkeit einer solchen paradigmatischen Kanalisierung bis hin zum Stereotyp.

Im Fall des Heimatkonzepts liegt aber diesbezüglich dennoch ein Sonderfall vor, insofern die Semantik von ,Heimat' auf der inhaltlichen Ebene genau diejenige Zentrierungs- und Normalisierungstendenz artikuliert, die auf der relationalen Ebene durch semantische Paradigmenstabilisierung formal und funktional hergestellt wird. ,Heimat' ist, anders gesagt, nicht ein Thema unter vielen, dessen Semantik im späten 19. Jahrhundert durch periodische Medien etabliert wird, sondern im Zuge dieser Etablierung gewissermaßen zugleich Metapher für diesen Prozess: Im Lichte fremder Länder und Kulturen folgt die Semantik von ,Heimat' derselben zentripetalen Kraft, die es einer Zeitschrift ermöglicht, trotz ihrer miszellanen Gestalt, die nicht zuletzt aus zahlreichen Berichten aus dem Ausland resultiert, ein einheitliches Programm zu vertreten.

Diese zweifach zentripetale Dimension des Heimatdiskurses beruht im Sinne der angesprochenen Wiederholungsstruktur auf der Konstellation einer Erzählung wie derjenigen von Herman Schmid mit den übrigen Texten im Heimgarten, die nach bzw. neben ihr erscheinen. Das betrifft vergleichbare Landschaftsschilderungen, wie sie sich etwa in Adolf Pichlers "Allerlei Geschichten aus Tirol" finden (Pichler 1877, 193), ${ }^{4}$ aber auch wiederkehrende Motive, die die Topik der Bedrohung des Heimatidylls fortsetzen, so z.B. die Artikelserie zu "Gift in Nahrungsmitteln" von Dr. R. Schilling, der die Todesart des Herzogs Franz Sigismund als aktuelle Gefahr präsentiert. Aber auch metanarrative Reflexionen der zentripetalen Effekte solcher Serienbildungen sind nachweisbar, so etwa wenn Pichler über Sagen schreibt, "dort verdichtet sich das sittliche Gefühl des Volkes zur mährchenhaften Erzählung" (Pichler

4 Pichler 1877, 193: „Ja, da trägt das Land seine Schützenfarben: Thal und Höhen überzieht üppiges Grün vom Blumenschmelz durchwoben, die Berge jedoch tragen den weitfaltigen, schneeweißen Mantel, während sich, noch dunkler, durch den Gegensatz, der Himmel tiefblau darüber wölbt." 
1877, 196). Und schließlich finden sich neben solchen affirmativen Diskursen durchaus auch ironische Brechungen, beispielsweise wenn wiederum Pichler die erste seiner Tiroler Geschichten beginnen lässt: „Der Frühling gehört in den Alpen durchschnittlich zur Regenzeit, so daß Kraut und Blumen sich eher darüber freuen mögen, als Touristen und Dichter" (Pichler 1877, 193).

$\mathrm{Zu}$ diesen Kontexten gehört auch Stifters Erzählung "Nachkommenschaften", die in der Nummer 6 des Heimgarten die Aufmacherposition von Schmids "Schütz" übernimmt. Die Stifter-Forschung hat den Text bislang immer nur für sich betrachtet und allenfalls seine Sonderstellung im Vergleich zu den übrigen Erzählungen des Autors hervorgehoben, die sich zum einen aus dem ungewöhnlich humoristischen Ton und zum anderen aus der ausdrücklichen Reflexion der Probleme des Realismus sowie des Epigonalismus ergibt (MeyerSieckendiek 2001; Hahn 2003; Oschmann 2009, 135-149). Liest man die "Nachkommenschaften" aber in Fortsetzung von "Der Schütz' von der Pertisau" sowie im Umfeld der übrigen Erzählungen im Heimgarten, so treten ganz andere Motivkomplexe in den Vordergrund, zu denen nicht zuletzt die ironische Decouvrierung von Schmids Projekt gehört, mit den Mitteln des Massenmediums Zeitschrift Heimat darzustellen.

Dass Stifters Erzählung innerhalb des Heimgarten eine eigenständige Rahmung gewinnt, erhellt bereits aus einigen Stifter-affinen Motiven in den Nachbartexten: Die Szene einer Rettung vor dem Gebirgsgewitter in "Der Schütz' von der Pertisau" wurde bereits erwähnt, und in den späteren Lieferungen von Pichlers "Allerlei Geschichten aus Tirol" tritt ein Mineraloge auf, der auf einer Wanderung in den Bergen auf "Katzensilber" und "bunte Steinchen" hingewiesen wir, weil er "für einen Maler gehalten" wird, der Alpenpanoramen für reiche Leute malt (Pichler 1877, 194). Eine Fortsetzungserzählung wie diejenige von Pichler weist damit nicht nur allgemeine intertextuelle Bezüge zu Stifters Erzählungen „Kazensilber" bzw. seine Erzählsammlung Bunte Steine von 1853 auf, sondern setzt im Heimgarten auch unmittelbar das Malermotiv aus den einige Nummern zuvor abgedruckten "Nachkommenschaften" fort.

Denn auch Stifters Erzählung handelt von einem Landschaftsmaler, der in den Alpen versucht, eine vollkommene Abbildung der Natur herzustellen. Zugleich reflektiert die Erzählung durch dieses Projekt aber auch das zeitschriftentypische Prinzip der Serialität, insofern der Erzähler eingesteht, anstelle eines gelungenen Werks eine vollständig unüberschaubare Reihe von Bildern zu produzieren, die als solche die ohnehin schon bestehende Menge von Landschaftsbildern ohne erkennbaren Qualitätsgewinn erweitere:

So bin ich unversehens ein Landschaftsmaler geworden. Es ist entsetzlich. Wenn man in eine Sammlung neuer Bilder geräth, welch' eine Menge von Landschaften gibt es da; wenn man in eine Gemäldeausstellung geht, welch' eine noch größere Menge von Landschaften trifft man da an, und wenn man alle Landschaften, welche von allen Landschaftsmalern unserer Zeit gemalt werden, von solchen Landschaftsmalern, die ihre Bilder verkaufen wollen,, und von solchen, die ihre Bilder nicht verkaufen wollen, ausstellte, welch' allergrößte Menge von Landschaften würde man da finden! (Stifter 1877a, 81)

Mein Vorschlag zu einer Lektüre dieser Eingangssätze im Kontext des Heimgarten besteht darin, sie als Kommentar zur Etablierung eines bestimmten Heimatbilds in Massenmedien - sowie zum Zusammenhang beider - zu lesen. Diese Lesart wird unterstützt durch Stifters Parallelisierung des Überhandnehmens von Landschaftsbildern durch dasjenige von Büchern:

\begin{abstract}
Oft, wenn ich die unzähligen Bücher betrachtete, welche sich in öffentlichen Sammlungen befinden, oder wenn ich die Verzeichnisse neugemachter Bücher ansah, dachte ich, wie man denn noch ein Buch machen kann, wenn schon so viele vorhanden sind; ja, wenn an eine neue erstaunliche Erfindung macht, so mag man selbe in einem Buche beschreiben und erklären; aber wenn man blos etwas erzählen will, da schon so unendlich Viele etwas erzählt haben, so erscheint das sehr überflüssig. (Stifter 1877a, 82)
\end{abstract}

Bezieht man diese Analogisierung von Bilder- und Büchermassen auch auf den zahlreichen Abdruck von Erzähltexten in Zeitschriften wie Der Heimgarten, dann wird zum einen der (selbstredend gezielt kalkulierte) performative Selbstwiderspruch der Feststellung, es sei sinnlos, weitere Beiträge zu den bereits bestehenden Massen an Erzählungen zu leisten, kenntlich, da diese Feststellung selbst in einer weiteren Zeitschriftenerzählung getroffen wird. Zum anderen wird man Zeitschriften selbst

5 Die Erstfassung ist auch greifbar in: Matz 2005, 13001360. 
zu denjenigen „öffentlichen Sammlungen" zählen können, in denen sich Texte ebenso wie Bilder wiederfinden - so dass auch die Produktion von Vorstellungen von Heimat nicht mehr auf Innerlichkeit und Lokalität, sondern auf Dispersion und Periodisierung beruht. Dann aber geht es im rahmenden Auftakt von Stifters Nachkommenschaften weniger um das allgemeine Problem der Originalität realistischer Kunstwerke, sondern vielmehr um eine satirische Perspektive auf den Umschlag der Repräsentation von Heimat in ein unüberschaubares Archiv von Texten bzw. Abbildungen:

\begin{abstract}
Nun, es sind der in Oelfarben gemalten und mit Goldrahmen versehenen Landschaften schon genug. Und ich will nun auch noch so viele Landschaften mit Oelfarben malen, als in mein noch übriges Leben hinein gehen. Ich bin jetzt sechsundzwanzig Jahre alt, mein Vater ist sechsundfünfzig, mein Großvater achtundachzig [...]; mein Urgroßvater, mein Ururgroßvater und deren Großväter und Ururgroßväter sind nach der Überlieferung der Großmutter über neunzig Jahre alt geworden: wenn ich nun auch so alt werde, und stets Landschaften male, so gehören, falls ich sie alle am Leben lasse, und sie einmal in Kisten sammt ihren Rahmen verpackt verführen will, fünfzehn zweispännige Wägen mit guten Rossen dazu [...]. (Stifter 1877a, 81)
\end{abstract}

Mit dieser satirischen Überspitzung der Vorstellung eines Lebenswerks bezieht sich der Erzähler auf ein konkretes Projekt, das auch den Handlungsraum der Erzählung im Anschluss an diese massenmedienreflexive Rahmung vorgibt: Der Erzähler berichtet von seinem Aufenthalt im "Lüpfinger Thale, an das mich auch eine Hexe gebannt hat. Es ist gar nicht schön, und hat ein langes Moor, von dem man das Fieber bekommt" (Stifter 1877a, 83). Damit ruft Stifter zum einen gängige Heimattopoi auf - Natur, Gesundheit und Aberglaube -, verkehrt sie aber alle in ihr Gegenteil. Und insofern das Moor Gegenstand des Malprojekts ist, ist auch das Motiv der massenhaften Repräsentation von Heimat von dieser Verkehrung betroffen:

[I]ch wollte Moor in Morgenbeleuchtung, Moor in Vormittagbeleuchtung, Moor in Mittagbeleuchtung, Moor in Nachmittagbeleuchtung beginnen [...]. Moor im Regen hatte ich mir schon vorgenommen, von meinem Fenster aus zu malen. Ueber das Moor im Nebel habe ich noch nicht nachgedacht. (Stifter 1877a, 85)

Zu dieser Verkehrung gängiger Elemente der Heimatsemantik gehört auch, dass das Malen des
Moors so dringlich ist, weil es im Zuge der ökonomischen Modernisierung trockengelegt werden soll - ein kaum verhohlenes Symbol der Bedrohung der Ursprünglichkeit der Natur:

Aber es ist nicht viel zu malen, denn da hat ein unbillig reicher Mann das Schloß Firnberg gekauft, und läßt so viele Steine und Erde in das Moor führen, und so viele Gräben von ihm hinwegziehen, daß das Moor kleiner und das Fieber weniger geworden ist. (Stifter 1877a, 83)

Und schließlich werden die Versatzstücke des Heimatbildes vollends dekonstruiert von der Tatsache, dass die Massenphänomene der medialen Repräsentation wie auch des später bei Pichler ebenfalls angesprochenen Tourismus die Begegnung mit der Alpenlandschaft um eine zweite Ebene erweitern, die jede Unmittelbarkeit verunmöglicht. Die Natur wird nicht mehr als solche, sondern als Modell wahrgenommen und ist entsprechen von Malern bevölkert:

Am Rande des Waldes dann, vor den Trümmern
eines alten Ritterschlosses, vor gethürmten Felsen,
vor gedehnten Ebenen, am Gestade des Meeres, in
Grotten und grünblauen Eishöhlen der Gletscher, vor
einzelnen Bäumen, Ruinen, Wässerlein, Waldpflanzen,
sind solche, welche sich bestreben, die Dinge, die sie
da sehen, mit Farben auf ihren Leinwanden zu bekom-
men. Dann macht noch ein Lehrer der Landschaftschule
von der Staatsmaleranstalt mit allen seinen Schülern
einen Ausflug, daß sie nun im Freien die Dinge gerade
so malen, wie sie sie sonst in der Stube nach seinen
Vorlagen gemalt haben. (Stifter 1877a, 82)

Und entsprechend wird auch der Erzähler gewarnt, sein Projekt, den Dachstein "treu und schön zu malen, als er ist", so "daß man den gemalten und den wirklichen nicht mehr zu unterscheiden vermöge", öffentlich bekannt zu machen: „Die Sache wird bekannt, die Zeitungen reden davon, Reisende kommen herzu, Engländer werden auf den Höhen herum sitzen, und mit Ferngläsern auf dein Häuschen schauen" (Stifter 1877, 82).

Stifters "Nachkommenschaften" nehmen mithin auf mehreren Ebenen auf Format und Programm ihres Erstpublikationsorts Bezug, wenn sie den Zusammenhang zwischen exzessivem Mediengebrauch, Popularisierung und dem Verlust der unberührten Natur inszenieren und reflektieren. Dadurch ist die Erzählung aber auch als deutlicher Gegenentwurf zu Herman Schmids affirmativem Heimatsnarrativ angelegt - eine Dimension, 
die sich erst durch die Wiederbesichtigung des Textes im Heimgarten erschließen lässt.

\section{VI}

Es gibt ein letztes Element dieses Heimatsnarrativs, das Stifter von Schmid übernimmt und in ein neues und zentrales Licht rückt: der Diskurs der Genealogie, der bei Schmid angesichts der ausbleibenden Fortsetzung der herzoglichen Linie ins Spiel kommt und Stifters Erzählung sogar den Titel leiht. "Nachkommenschaften" meint dabei zwar zunächst den bildkünstlerischen Nachlass des Erzählers, dann aber auch seine Familienstruktur, und beide Bereiche des Vererbens werden im Text zunehmend verknüpft (Willer 2009, 45-62). ${ }^{6}$ So beruht die Lösung des Archivierungsproblems des bildkünstlerischen Nachlasses eines Malers, der nicht - wie aus ästhetischen Gründen geboten - seine alten Bilder vernichtet, wenn er neue, bessere malt (so dass „bei meinem Tode nur ein Bild von mir vorhanden" wäre; Stifter 1877a, 83), auf der Hoffnung, dass er eine große Zahl von "Nichten, Neffen, Geschwisterkinder, Urnichten, Urneffen, Urgeschwisterkinder, Ururnichten Ururneffen, Ururgeschwisterkinder, und so weiter, in großer Zahl haben werde, unter welchen ich meine Bilder als Geschenke vertheilen kann" (Stifter 1877a, 83). Zu dieser Verbindung zwischen Malerei und Genealogie, tritt die bereits bei Schmid etablierte zwischen Genealogie und Heimat. Denn das Ziel des erwähnten Projekts des "reichen Mannes", das Moor im Lüpfinger Tal trockenzulegen, besteht darin, seiner Familie einen gemeinsamen Lebensort zu erschaffen:

Es ist stets ein merkwürdiges Zeichen der Roderer gewesen, daß sie immer in der Welt zerstreut waren, keiner Gegend angehörten, bald hier bald da auftauchten, und wieder verschwanden, es gehört dieß zu ihrem begabten oder unstäten Wesen, und mehrt dieses Wesen hinwiederum. Ich möchte eine festen Stamm der Roderer in dieser Gegend gründen, und ihn an diese Gegend heften, und wenn meine Nachkommen so denken wie ich, so trocknen sie das Moor völlig aus, verwalten ihre liegende Habe, genießen das Erworbene, vermindern es nie, vermehren es

6 Vgl. zur natürlich-kulturellen Doppelsemantik des Begriffs der Vererbung sowie des zugehörigen Verständnisses von Genealogie Parnes/Vedder/Weigel/Willer 2005. dagegen, wirken gut für die Menschen hier, verwachsen mit ihnen, werden stätig und ruhig, bleiben stets bürgerlich, und sagen: Peter Roderer der Amsterdamer ist der erste gewesen, der sich hier ansässig gemacht hat. (Stifter 1877b, 100)

Der Erzähler erkennt sich in diesem Prospekt mehrfach wieder: Er sieht den Roderers nicht nur hinsichtlich Augenfarbe und Barttracht auffällig ähnlich, er trägt denselben Nachnamen ${ }^{7}$ und stammt seinerseits aus einer Familie, die über keine gemeinsame Heimat verfügt:

Mein Vater ist erst von Siebenbürgen nach Wien übergesiedelt; der eine Oheim wohnt in Mähren, der andere ist auch erst kurz in Wien und der Großoheim hat die vielen Hasen alle in Schlesien geschossen. Ich selber bin noch gar kein Ansässiger [...]. (Stifter 1877b, 101)

All dies erweist sich im Fortgang der Erzählung nicht als "Zufall", sondern als tatsächliche Verwandtschaft zweier Zweige der Roderer, und als der junge Maler sich in die Tochter des alten Mannes verliebt und seine Kunst zugunsten einer Heirat aufgibt, kommt es anlässlich der Hochzeit zur "Stammesverbrüderung" aller Roderer, deren "Stammbaum nun aus den Erinnerungen meines Vaters und Roderers völlig aufgestellt worden" (Stifter 1877c, 117).

Indem der Erzähler im Vorfeld dieser Hochzeit seine Bilder zerstört, um sich ganz dem Familienleben zu widmen, führen die „Nachkommenschaften" vor, wie der Ansatz, Heimat durch die Darstellung von Landschaft zu vermitteln, durch ein genealogisches Verständnis von Heimat ersetzt wird - und das umso mehr, als die Familienzusammenführung und -fortführung in

7 Der Name Roderer ist dabei mit Blick auf die Ansiedlung und Heimatbildung ebenso sprechend, wie der Autorname Stifter, mit dessen lateinischer Form fundator etwa in der Erstfassung von „Die Mappe meines Urgroßvaters" gespielt wird. Entsprechend hat sich Stifter in einem Brief an seine Frau auch mit der Figur des alten Roderer identifiziert, und auch das Projekt eines exakten Abbilds des Dachstein ist autobiographisch fundiert. Wenn in "Nachkommenschaften" die Wirtin auf die Namensgleichheit des Erzählers mit dem alten Roderer hin weitere in der Gegend übliche Namen aufzählt - „Bei uns sind viele Meier, Bauer, Schmid." (Stifter 1877a, 84) -, so gewinnt das im Kontext der Publikation im Heimgarten insofern eine zusätzliche Dimension, als Stifter hier den Nachnamen des Herausgebers einreiht und sogar in Relation zu seinem Rollennamen setzt: ,',So, Meier, Bauer, Schmid, 'sagte ich, ,diese gemeinen Dinge; aber Roderer!" (Stifter 1877a, 84). 
Gestalt der Hochzeit ja einen exakten Gegenentwurf zum tragischen Scheitern dieses Vorhabens in "Der Schütz' von der Pertisau" darstellt, wo der genealogische Plan durch die Intrige der Italiener gerade nicht aufgeht. Wenn die Festgesellschaft aber am Ende auf "Das Doppelrodererwohl auf grenzenlose Zeit!" (Stifter 1887c, 118) anstößt, dann liest sich das nicht zwingend wie all die anderen harmonischen Schlusswendungen in Stifters Romanen und Erzählungen, für die der Autor bekannt, wenn nicht gefürchtet ist. Durchaus schwingt in dem genealogischen Kompositum „Doppelroderer" eine ironische Brechung des Paradigmenwechsels von der Heimatrepräsentation zur genealogischen Heimat mit. Denn Stifter entwirft mit den "Nachkommenschaften" nicht nur eine Meta-Erzählung über die unterschiedlichen Paradigmen von Heimatdiskursen, wie sie sich in den einschlägigen Zeitschriften finden, sondern führt vor, auf welche Weise auch das genealogische Paradigma in den Paradoxien des repräsentierenden gefangen bleibt: Denn insofern die Verheiratung der beiden Roderer-Zweige in die Versammlung aller Verwandten und Nachkommen mündet, entwirft das Ende der Erzählung ein Tableau, das ebenso quantitativ überbordend und verwirrend ist, wie die Zahl der Gemälde und Bücher zu Beginn. Stifter reflektiert in den "Nachkommenschaften" also nicht nur, wie der Heimatdiskurs der Zeitschriftenpresse zu einer unaufhaltsamen Serie von Texten und Bildern führt, sondern dass auch die bei Schütz noch utopisch projizierte genealogische Kontinuität ebenfalls in inflationäre Unüberschaubarkeit mündet.

Die Familie der Roderer ist dabei als wiederhergestellte Einheit aus der Differenz bzw. Wiederansiedlung und Wiederzusammenführung des Zerstreuten und Heterogenen genau so paradox verfasst, wie die Semantik von Heimat einerseits und die Erscheinungsform von Zeitschrift, in denen diese Semantik produziert wird, andererseits: So, wie das Konzept , Heimat' nicht auf eine vorgegebene Einheit von Raum und Familie rekurrieren kann, sondern diese Bezugspunkte durch Landgewinnung und Verwandtenheirat allererst ex post herstellen muss, ist auch das Medium, das dieser Herstellung von Heimat dient, eines, dass seine Einheit gegenüber der faktischen Miszellanität und Serialität periodischen Publizierens immer wieder behaupten muss.
Es geht mit anderen Worten in "Nachkommenschaften" immer auch um das Paradox, eine zentripetale Semantik von Heimat in einem zentrifugalen Medienformat zu entwerfen - ein Bezug, der allerdings nur dann kenntlich wird, wenn man die Erzählung selbst als Beitrag zu einer Heimatzeitschrift wiederliest. Innerhalb dieses Kontexts führt der Text aber besonders anschaulich vor, auf welche Weise ,Heimat' bereits im 19. Jahrhundert als massenmediales Konstrukt reflektiert wurde, dessen Referenz nicht in der konkreten Präsenz einer Landschaft oder Genealogie einer Familie besteht, sondern im Imaginären einer kollektiven Rezeption vermischter und zerstreuter Texte - also im Imaginären des Mediums Zeitschrift selbst.

\section{Literaturverzeichnis}

Anderson, Benedict (2006): Imagined Communities. Reflections on the Origin and Spread of Nationalism. London/New York: verso.

Beetham, Margaret (1990): Towards a Theory of the Periodical as a Publishing Genre. In: Brake, Laurel/ Jones, Aled/Madden, Lionel (Hgg.): Investigating Victorian Journalism. New York: Palgrave Macmillan, S. 19-32.

Derrida, Jacques (1974): Grammatologie. Frankfurt/M.: Suhrkamp.

Farkas, Rainhart (2000): Roseggers Heimgarten Literarische Texte im Spannungsfeld übergreifender Dispositive. Lebensform - Heimatdiskurs - Reagrarisierung. In: Panagl, Oswald/ Weiss, Walter (Hgg.): Noch einmal Dichtung und Politik. Vom Text zum politisch-sozialen Kontext und zurück. Wien: Böhlau, S. 177-193.

Gebhard, Gunther/Geisler, Oliver/Schröter, Steffen (Hgg.) (2007): Heimat. Konturen und Konjunkturen eines umstrittenen Konzepts. Bielefeld: transcript.

Gretz, Daniela (2016a): Poetik der Miszelle? Präliminarien zur Koevolution von periodischer Presse und modernem Roman mit Blick auf Raabes Stopfkuchen und einem Ausblick auf Fontanes Stechlin. In: Colloquia Germanica 49, Themenheft "Periodical Literature in the Nineteenth Century", S. 305-328.

Gretz, Daniela (2016b): „Eine große Zeitungsthat": Die serielle Exploration des I/inneren Afrika/s in populären Zeitschriften des 19. Jahrhunderts. In: Gretz, Daniela/Pethes, Nicolas (Hg.): Archiv/ Fiktionen. Verfahren des Archivierens in Literatur und Kultur des langen 19. Jahrhunderts. Freiburg: Rombach, S. 279-315.

Hahn, Marcus (2003): Geschichte und Epigonen. ,19. Jahrhundert'/,Postmoderne', Stifter/Bernhard. Freiburg: De Gruyter. 
Hamann, Christof: Zwischen Normativität und Normalität. Zur diskursiven Position der ,Mitte' in populären Zeitschriften nach 1848. Heidelberg: Synchron 2014. Kaminski, Nicola/Ramtke, Nora/Zelle, Carsten (Hgg.) (2014): Zeitschriftenliteratur/Fortsetzungsliteratur. Hannover: Wehrhahn.

Kaminski, Nicola/Ruchatz, Jens (2017): Journalliteratur - ein Avertissement. Pfennig-Magazin zur Journalliteratur, Heft 1, Hannover: Wehrhahn.

Lipp, Wilfried (1986): Heimatbewegung, Regionalismus - Pfade aus der Moderne? In: Kölner Zeitschrift für Soziologie und Sozialpsychologie, Sonderheft 27, S. 331-355.

Meyer-Sieckendiek, Burkhard (2001): Die Ästhetik der Epigonalität. Theorie und Praxis wiederholenden Schreibens im 19. Jahrhundert: Immermann - Keller - Stifter - Nietzsche. Tübingen: A. Francke.

Matz, Wolfgang (Hg.) (2005): Adalbert Stifter: Sämtliche Erzählungen nach den Erstdrucken. München: dtv.

Mussell, James (2012): The Nineteenth-Century Press in the Digital Age. New York: Palgrave Macmillan.

Mussell, James (2015): Repetition, or „In Our Last”. In: Victorian Periodicals Review 48, S. 343-358.

Nitzke, Solvejg (2018): Zurück zur Natur. Erfindung und Verschwinden der Waldheimat. In: Weiland, Marc/Ehrler, Martin (Hgg.): Topografische Leerstellen - Ästhetisierungen verschwindender und verschwundener Dörfer und Landschaften in Literaturen, Filmen und Künsten, Bielefeld: transcript, S. $199-214$.

Oschmann, Dirk (2009): Absolute Darstellung. Zur Metapoetik von Stifters Nachkommenschaften. In: Literaturwissenschaftliches Jahrbuch 50, S. 135-149.

Parnes, Ohad/Vedder, Ulrike/Weigel, Sigrid/Willer, Stefan (Hgg.) (2005): Generation. Zur Genealogie des Konzepts - Konzepte von Genealogie. München: Wilhelm Fink.
Pichler, Adolf (1877): Allerlei Geschichten aus Tirol, Teil 1. In: Der Heimgarten 1, Nr. 13, S. 193-200.

Radkau, Joachim (2011): Die Ära der Ökologie. Eine Weltgeschichte, München: C. H. Beck.

Rosegger, Peter (Hg.) (1877): Heimgarten. Eine Monatsschrift, Heft 1.

Schmid, Herman (1877a): Der Schütz' von der Pertisau, Teil 2. In: Der Heimgarten 1, Nr. 2, S. 2-29.

Schmid, Herman (1877b): Der Schütz' von der Pertisau, Teil 3. In: Der Heimgarten 1, Nr. 3, S. 33-40.

Sieferle, Rolf-Peter (1984): Fortschrittsfeinde? Opposition gegen Technik und Industrie von der Romantik bis zur Gegenwart. München: C. H. Beck.

Stifter, Adalbert (1877a): Nachkommenschaften, Teil 1. In: Der Heimgarten 1, Nr. 6, S. 81-88.

Stifter, Adalbert (1877b): Nachkommenschaften, Teil 2. In: Der Heimgarten 1, Nr. 7, S. 97-110.

Stifter, Adalbert (1877c): Nachkommenschaften, Teil 8. In: Der Heimgarten 1, Nr. 8, S. 113-118.

Stockinger, Claudia (2018): An den Ursprüngen populärer Serialität. Das Familienblatt Die Gartenlaube. Göttingen: Wallstein.

Twellmann, Marcus (2019): Dorfgeschichten. Wie die Welt zur Literatur kommt. Göttingen: Wallstein.

Adam, Thomas (1998): Die Verteidigung des Vertrauten. Zur Geschichte der Natur- und Umweltschutzbewegung in Deutschland seit Ende des 19. Jahrhunderts. In: Zeitschrift für Politik NF 45, S. 20-48.

Willer, Stefan (2009): Grenzenlose Zeit, schlingender Grund. Genealogische Ordnungen in Stifters Nachkommenschaften. In: Gamper, Michael/Wagner, Karl (Hgg.): Figuren der Übertragung. Adalbert Stifter und das Wissen seiner Zeit. Zürich: Chronos, S. $45-62$. 